\title{
Neutrino Astronomy with IceCube
}

\author{
Kevin J. Meagher ${ }^{1}$ on behalf of the IceCube Collaboration ${ }^{2}$ \\ ${ }^{1}$ Université Libre de Bruxelles, Science Faculty CP230, \\ B1050 Bruxelles, Belgium \\ email: kmeagher@ulb.ac.be \\ ${ }^{2}$ website: http://icecube.wisc.edu
}

\begin{abstract}
The IceCube Neutrino Observatory is a cubic kilometer neutrino telescope located at the Geographic South Pole. Cherenkov radiation emitted by charged secondary particles from neutrino interactions is observed by IceCube using an array of 5160 photomultiplier tubes embedded between a depth of $1.5 \mathrm{~km}$ to $2.5 \mathrm{~km}$ in the Antarctic glacial ice. The detection of astrophysical neutrinos is a primary goal of IceCube and has now been realized with the discovery of a diffuse, high-energy flux consisting of neutrino events from tens of $\mathrm{TeV}$ up to several $\mathrm{PeV}$. Many analyses have been performed to identify the source of these neutrinos: correlations with active galactic nuclei, gamma-ray bursts, and the galactic plane. IceCube also conducts multimessenger campaigns to alert other observatories of possible neutrino transients in real-time. However, the source of these neutrinos remains elusive as no corresponding electromagnetic counterparts have been identified. This proceeding will give an overview of the detection principles of IceCube, the properties of the observed astrophysical neutrinos, the search for corresponding sources (including real-time searches), and plans for a next-generation neutrino detector, IceCube-Gen2.
\end{abstract}

Keywords. acceleration of particles, neutrinos, galaxies: active, gamma rays: bursts

\section{Neutrino Astronomy}

From radio waves to gamma-rays, electromagnetic radiation has been the source of a wealth of information about the universe. Unfortunately, photons with energies above $1 \mathrm{TeV}$ are absorbed on the extra-galactic background light making it impossible to see extra-galactic sources at these energies. In order to study the universe above this cut-off we need to find an alternative to photons. Cosmic rays tell us that charged particles are accelerated by astrophysical objects up to at least $10^{20} \mathrm{eV}$, but since charged particles are deflected by magnetic fields, the origin of these particles still remains unclear. Since neutrinos interact solely via the weak force, they can traverse the universe completely unimpeded and therefore hold the potential to open a new window on astronomy.

\section{The IceCube Neutrino Observatory}

Neutrinos' small cross-section, the same property that allows them to arrive at Earth unimpeded, also makes them difficult to detect. Observing neutrinos requires a large target mass to make up for the small cross-section. In addition, the medium must be transparent in order to observe the light from the secondary particles. The IceCube Neutrino Observatory was built in the Antarctic glacier under the South Pole Station.

The fundamental unit of IceCube is the digital optical module (DOM). Each DOM contains a $25 \mathrm{~cm}$ photomultiplier tube, high voltage power supply, and digitization and communication electronics. The 60 DOMs are aligned on vertical structures called strings, with a vertical spacing of $17 \mathrm{~m}$ between a depth of $1450 \mathrm{~m}$ and $2450 \mathrm{~m}$. There are 86 
strings for a total of 5160 DOMs. The strings form a triangular grid with a spacing of $125 \mathrm{~m}$, except for 8 strings arrayed in the center to form a denser formation referred to as DeepCore.

There are three main detector channels used for neutrino astronomy: muon tracks, cascades, and HESE. Muon tracks are produced from charged current $\nu_{\mu}$ interactions. At these energies, muons can travel tens of kilometers from the interaction vertex. The observed $1 \mathrm{~km}$ tracks allow for muons to be reconstructed with good angular resolution, $0.7^{\circ}$ for energies above $10 \mathrm{TeV}$. Including muons produced far from the detector greatly increases the effective area of this channel. The energy that the muon deposits in the detector can be measured, but this may be significantly less than the energy of the original neutrino. However, it is difficult to distinguish neutrinos from muon bundles produced by cosmic ray air showers, so the track channel has sensitivity primarily in the Northern Hemisphere where air shower bundles are screened out by the Earth.

The cascade channel uses events from either neutral current interactions or charged current interactions from electron and tau neutrinos. These interactions create secondary particles which lose energy on length scales much smaller than the spacing of the strings and thus appear to be near point-like emissions of light from the interaction vertex. Since all the energy is deposited near the vertex, these events have much better energy resolution than tracks, but at the cost of relatively poor angular resolution.

The high energy starting-event (HESE) channel observes events which start in the detector volume, by only selecting events where the initial light occurs on DOMs within the interior of the detector, and vetoing events which start near the edge. Although the events in this channel may be considered either tracks or cascades, from an analysis point of view it is often easier to consider HESE as a separate channel.

IceCube experiences two main kinds of background: muon bundles from cosmic ray air showers referred to simply as muons and neutrinos from the same air showers which are referred to as atmospheric neutrinos. Muons are only a background in the Southern Hemisphere as the Earth screens out muons from the Northern Hemisphere. But atmospheric neutrinos represent an irreducible background which can only be distinguished from astrophysical neutrinos by their energy spectrum. The atmospheric neutrino spectrum is very soft, being described with a power-law of $E^{-3.7}$ or steeper while acceleration mechanisms for astrophysical objects are expected to produce a spectral index of 2-2.5.

\section{Observation of High-Energy Neutrinos}

Using the HESE channel, an analysis performed on 4 years of data found 54 neutrino candidate events with a statistical significance of $6.5 \sigma$ (Aartsen et al. 2015a). In order to describe the data, a maximum likelihood, forward-folding fit of all components (atmospheric muons, atmospheric neutrinos from $\pi / \mathrm{K}$ decay, atmospheric neutrinos from charm decay and an astrophysical flux assuming a 1:1:1 flavor ratio) was performed on the energy spectrum. The result of the fit, shown in Figure 1 (left), is $\mathrm{d} N / \mathrm{d} E=(2.2 \pm 0.7) \times 10^{-18} \cdot(E / 100 \mathrm{TeV})^{-2.58 \pm 0.25} \mathrm{GeV}^{-1} \mathrm{~cm}^{-2} \mathrm{~s}^{-1} \mathrm{sr}^{-1} \cdot$ A maximum likelihood clustering method was used to look for any neutrino point sources in this sample. This test, shown in Figure 1 (right), did not yield significant evidence of clustering with p-values of $44 \%$ and $58 \%$ for the shower-only and the all-events tests, respectively. A test for galactic plane clustering was also performed. Assuming a galactic width of $2.5^{\circ}$ around the plane resulted in a p-value of $7 \%$ and a variable galactic width scan resulted in a p-value $2.5 \%$.

A separate diffuse spectral analysis was performed using six years of data with the muon track channel (Aartsen et al. 2016a). At energies between $191 \mathrm{TeV}$ and $8.3 \mathrm{PeV}$ a 

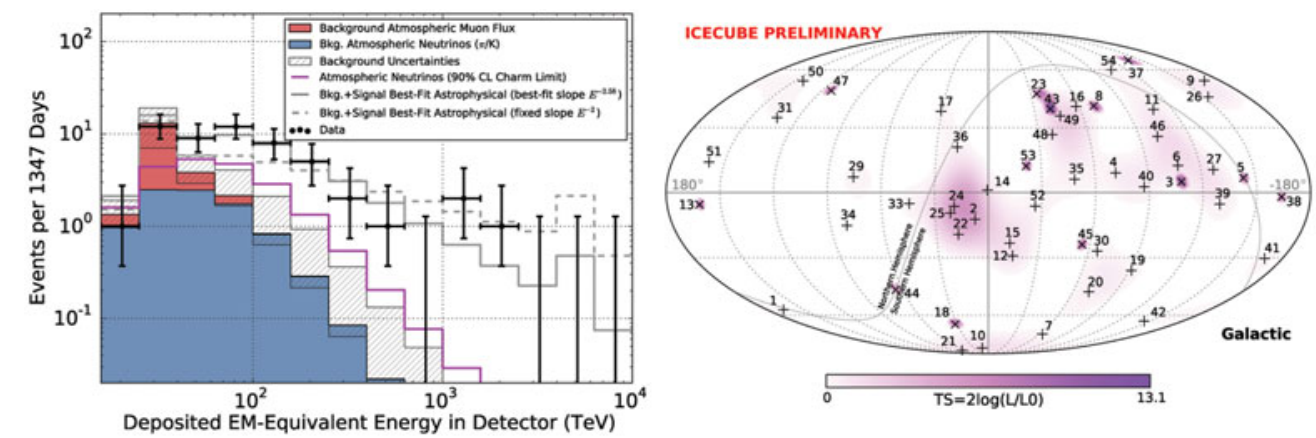

Figure 1. Left: Deposited energy spectrum of high energy starting-event (HESE) channel (Aartsen et al. 2015a). The hashed region shows uncertainties on the sum of all backgrounds. Muons are computed from simulation to overcome statistical limitations in our background measurement and scaled to match the total measured background rate. Atmospheric neutrinos and uncertainties thereon are derived from previous measurements of both the $\pi / \mathrm{K}$ and charm components of the atmospheric $\nu_{\mu}$ spectrum. Right: Arrival directions of the same sample in galactic coordinates. Shower-like events are marked with "+" and those containing tracks with " $\times$ ". Colors show the test statistics for the point-source clustering test at each location. No significant clustering was found.
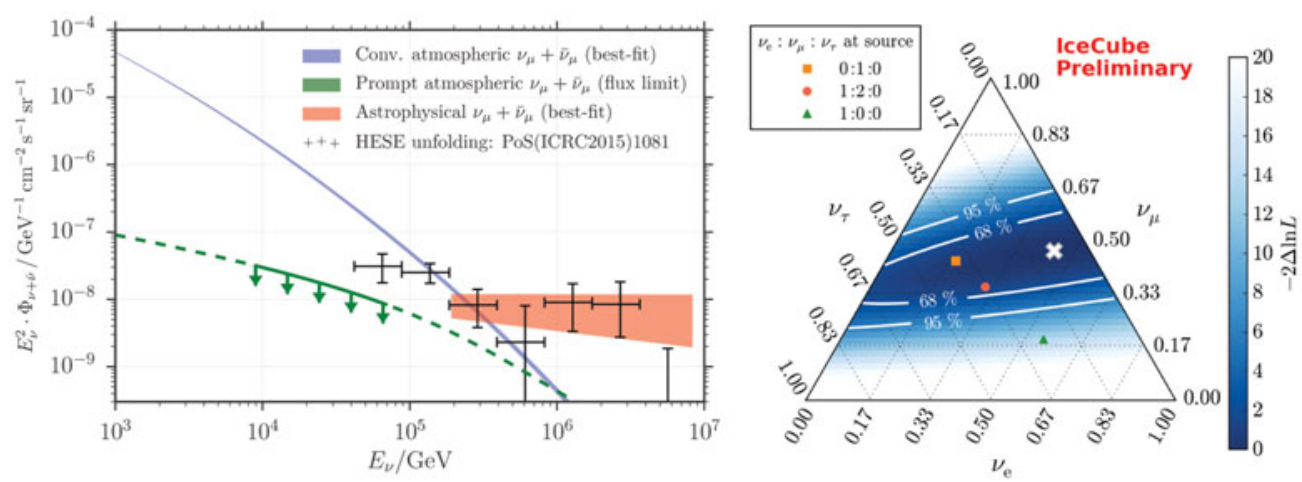

Figure 2. Left: Best-fit neutrino spectra for the unbroken power-law model using muon neutrino interactions. The conventional and astrophysical neutrino fluxes are represented by shaded regions indicating one sigma error on the measured spectrum where as the solid line represents the upper limit on the prompt neutrino model in Enberg et al.(2008). The horizontal width of the astrophysical shaded region denotes the range of neutrino energies which contribute $90 \%$ to the total likelihood ratio between the best-fit and the conventional atmospheric-only hypothesis. The crosses show the unfolded spectrum of the high-energy sample discussed above. Right: The results of the profile likelihood scan of the flavor composition at Earth. Each point in the triangle corresponds to a ratio $\nu_{e}: \nu_{\mu}: \nu_{\tau}$ as measured on Earth, the individual contributions are read off the three sides of the triangle. The best-fit composition is marked with " $\times$ ", $68 \%$ and $95 \%$ confidence regions are indicated. The ratios corresponding to three flavor composition scenarios at the sources of the neutrinos are marked by the square for pion-decay $(0: 1: 0)$, circle for muon-damped (1:2:0), and triangle for neutron-beam (1:0:0) sources respectively.

significant astrophysical contribution was observed with a significance of $5.6 \sigma$. As shown in Figure 2 (left), the data was well-described by a power-law: $\mathrm{d} N / \mathrm{d} E=\left(0.90_{-0.27}^{+0.30}\right) \times$ $10^{-18} \cdot(E / 100 \mathrm{TeV})^{-2.13 \pm 0.13} \mathrm{GeV}^{-1} \mathrm{~cm}^{-2} \mathrm{~s}^{-1} \mathrm{sr}^{-1}$. Compared to HESE, this result has a harder spectrum. However, this analysis has a lower energy threshold, possibly indicating a break in the astrophysical neutrino spectrum. This analysis included the detection of a track with a reconstructed muon energy of $4.5 \pm 1.2 \mathrm{PeV}$ which implies a probability of less than $0.005 \%$ for this event to be of atmospheric origin. In addition, this analysis 


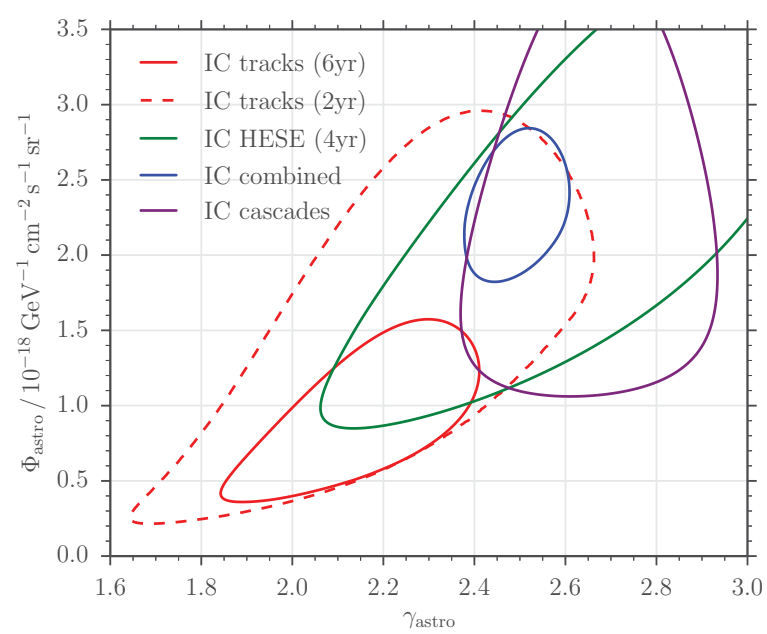

Figure 3. Results of different IceCube analyses measuring the astrophysical flux parameters $\Phi_{\text {astro }}$ and $\gamma_{\text {astro }}$. The contour lines show the $90 \%$ CL. The result of the muon track diffuse analysis (Aartsen et al. 2016a) is shown by the bottom-left solid contour line. The contour obtained by the previous measurement using the same method but using 2 years of data is the bottom-left dashed line. The results of the HESE analysis (Aartsen et al. 2015a), cascade channel (Aartsen et al. 2015b), and the combined analysis (Aartsen et al. 2016a) are also shown.

was able to constrain the contribution from a prompt atmospheric muon neutrino flux originating from charmed meson decays below 1.06 of the flux normalization from Enberg et al. (2008).

The ratio of different neutrino flavors can give important clues to acceleration mechanisms of the source. In Aartsen et al.(2015b) we also performed a measurement of the flavor composition of the astrophysical neutrino flux, in which the normalizations of all three flavors were allowed to vary independently. The results, shown in Figure 2 (right), are consistent with pion-decay sources and muon-damped sources but disfavor neutronbeam sources with a significance of $3.6 \sigma$.

In the cascade channel, in an analysis of the first two years of data a total of 172 events were observed with energies between $10 \mathrm{TeV}$ and $1 \mathrm{PeV}$ (Aartsen et al. 2015b). The astrophysical component is also well described by a power-law: $\mathrm{d} N / \mathrm{d} E=\left(2.3_{-0.6}^{+0.7}\right) \times$ $10^{-18} \cdot(E / 100 \mathrm{TeV})^{-2.67 \pm 0.13} \mathrm{GeV}^{-1} \mathrm{~cm}^{-2} \mathrm{~s}^{-1} \mathrm{sr}^{-1}$. The background-only hypothesis is rejected with a significance of $4.7 \sigma$.

The results of these analyses along with the results of 3 other diffuse analyses were combined into a global spectral analysis (Aartsen et al. 2016a). Assuming the astrophysical neutrino flux to be isotropic and to consist of equal flavors at Earth, the all-flavor spectrum with neutrino energies between $25 \mathrm{TeV}$ and $2.8 \mathrm{PeV}$ is well described by an unbroken power law with a best-fit spectral index $2.50 \pm 0.09$ and a flux at $100 \mathrm{TeV}$ of $6.7_{-1.2}^{+1.1} \cdot 10^{-18} \mathrm{GeV}^{-1} \mathrm{~s}^{-1} \mathrm{sr}^{-1} \mathrm{~cm}^{-2}$. Under the same assumptions, an unbroken power law with index 2 is disfavored with a significance of $3.8 \sigma(\mathrm{p}=0.0066 \%)$ with respect to the best fit. This significance is reduced to $2.1 \sigma(\mathrm{p}=1.7 \%)$ if instead we compare the best fit to a spectrum with index 2 that has an exponential cut-off at high energies. Allowing the electron neutrino flux to deviate from the other two flavors, we find a $\nu_{e}$ fraction of $0.18 \pm 0.11$ at Earth. The sole production of anti-electron neutrinos, which would be characteristic of neutron-decay dominated sources, is rejected with a significance of $3.6 \sigma(\mathrm{p}=0.014 \%)$. The results of the combined sample spectral fit along with the previously mentioned analyses are shown in Figure 3. Significant tension is seen between the 

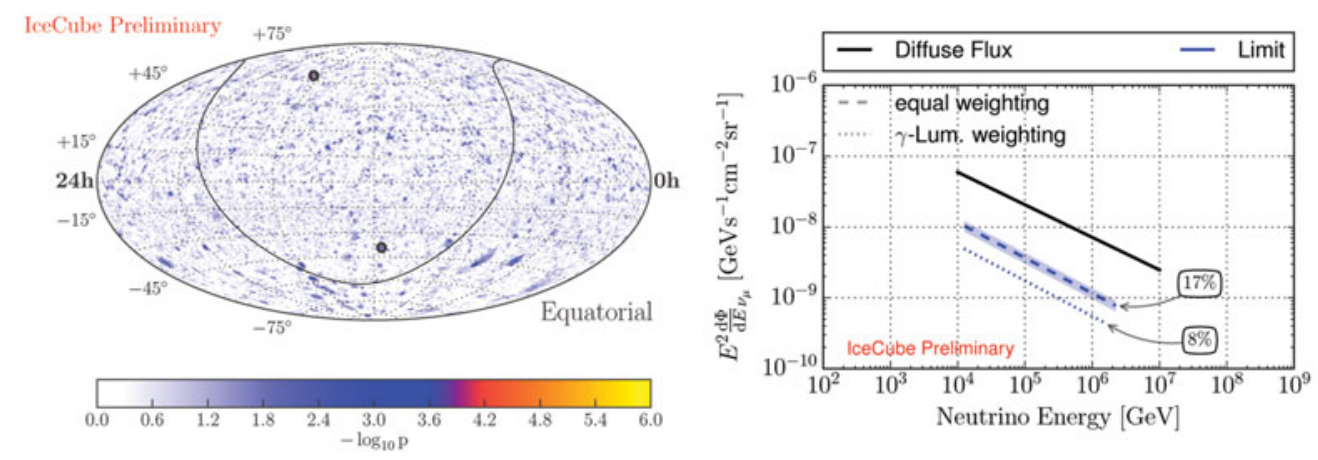

Figure 4. Left: Pre-trial significance skymap of the all-sky point source scan (Aartsen et al. 2015d). The color indicates the negative logarithm of the pre-trial p-value assuming no clustering as null-hypothesis. Shown in Equatorial (J2000) coordinates, a line indicates the galactic plane. The most significant fluctuation in each hemisphere is marked. Right: Results of the stacked blazar analysis. Neutrino flux upper limits for an $E^{-2.5}$ spectrum from Glüsenkamp (2016) compared to diffuse bestfit (solid line) from Aartsen et al. (2016a). Two seperate signal weighting schemes are shown: equal weighting (dashed line) where blazars are considered to contribute equally to the neutrino flux and weighitng by blazars' observed gamma-ray luminosity (dotted line).

different analyses. Since each analysis has a different sensitivity as a function of energy and zenith angle, the difference may be due to the energy spectrum changing shape at higher energy or possibly due to a population of harder sources in the Southern Hemisphere.

\section{The Search for Astrophysical Sources}

To identify the source of the neutrino populations described in the previous section, many analyses have been performed. To date none of them have identified any association with known or unknown astrophysical sources. In seven years of data, from 2008-2015, using an unbinned maximum-likelihood search for local clustering in the muon channel, no significant clustering of neutrinos above background expectation was observed (Aartsen et al. 2015d). The map generated by this analysis is shown in Figure 4 (left). The negative result of this analysis excludes point sources with a flux of $E^{2} \mathrm{~d} \Phi / \mathrm{d} E=10^{-12} \mathrm{TeV} \mathrm{cm}^{-2} \mathrm{~s}^{-1}$.

Blazars have been proposed as a possible source of high-energy neutrinos. To investigate this a stacked analysis was performed with blazars from the 2nd Fermi-LAT AGN catalogue (2LAC) (Glüsenkamp 2016). No significant excess is observed, constraining the total population of $2 \mathrm{LAC}$ blazars to contributing $27 \%$ or less of the observed astrophysical neutrino flux, assuming equipartition of neutrino flavors at Earth and the currently favoured power-law spectral index for the neutrino flux of 2.5. As shown in Figure 4 (right), the 2LAC blazars (and sub-populations) are excluded as being the dominant sources of the observed neutrinos up to a spectral index as hard as 2.2.

Another astrohphysical source considered to be a likely source of neutrinos are gammaray bursts (GRBs). An analysis incorporating 4 years of muon track events and 506 observed Northern Hemisphere bursts found no correlation more significant than expected from background (Aartsen et al. 2015c). In addition, a 3 year search using the cascade channel correlated with $807 \mathrm{GRBs}$ from the whole sky found similar results (Aartsen et al. 2016b). The limits on the neutrino flux set by these analyses (see Figure 5 ,left) disfavor much of the parameter space for the theories on neutrino emission from 

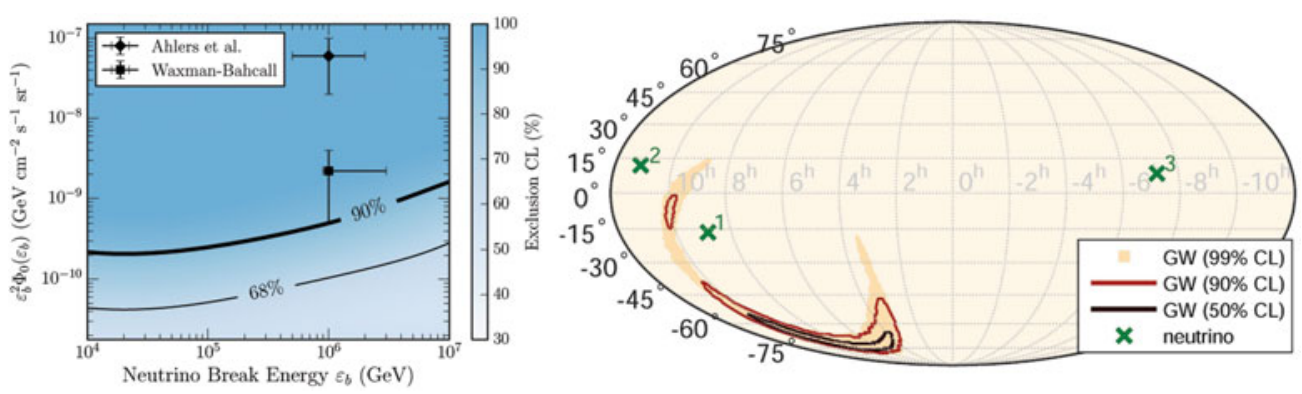

Figure 5. Left: Constraint on generic doubly broken power law neutrino flux models as a function of first break energy $\varepsilon_{b}$ and normalization $\Phi_{0}$. The model by Ahlers et al. (2011) assumes that only neutrons escape from the GRB fireball to contribute to the UHECR flux. The Waxman \& Bahcall model (1997), which allows all protons to escape the fireball, has been updated to account for more recent measurements of the UHECR flux (Katz et al. 2009) and typical gamma break energy (Goldstein et al. 2012). Exclusion contours, calculated from the combined 4 year all-sky analysis (Aartsen et al. 2016b) with the 4 year Northern Hemisphere analysis (Aartsen et al. 2015c), are shown. Right: Gravitational wave skymap in equatorial coordinates, showing the reconstructed probability density contours of the $\mathrm{GW}$ event at $50 \%$, $90 \%$ and $99 \% \mathrm{CL}$, and the reconstructed directions of high-energy neutrino candidates detected by IceCube (crosses) during a $\pm 500 \mathrm{~s}$ time window around gravitational wave event GW150914. The neutrino directional uncertainties are $<1^{\circ}$ and are not shown. Gravitational wave shading indicates the reconstructed probability density of the gravitaitonal wave event, darker regions corresponding to higher probability.

GRBs. Combined, these analyses find that no more than $1 \%$ of the observed astrophysical neutrino flux consists of prompt emission from GRBs that are observable by existing satellites.

Another considered source was the first gravitational wave transient GW150914 observed by the Advanced LIGO detectors on Sept. 14th, 2015. The analysis was perfomed by looking for neutrino candidates within $500 \mathrm{~s}$ of the gravitational wave event. As shown in Figure 5 (right) and consistent with background, 3 events were observed within this time window, none of them within the region triangulated by LIGO (Adrián-Martánez et al. 2016).

In order to alert other astronomers about possible neutrino transient events, the IceCube collaboration has developed several real-time alert programs. The neutrino data is processed in real-time at the South Pole station and the most interesting neutrino events are selected to trigger observations with optical and X-ray telescopes aiming for the detection of an electro-magnetic counterpart such as a GRB afterglow or a rising SN light curve. The program is capable of triggering follow-up observations in less than a minute. The optical follow-up program (Abbasi et al. 2012) has been sending such alerts to optical telescopes since 2008 and to X-ray telescopes since 2009. The gamma-ray follow-up program has been running since 2012 (Aartsen et al. 2016c), sending triggers to the MAGIC and VERITAS gamma-ray telescopes. This program focuses on blazar flares by monitoring a predefined list of known blazars and looks for excesses of neutrino events on timescales of up to three weeks.

\section{Future Instrumentaion}

Although IceCube has positivetly identified neutrinos of astrophysical origin, the ability of IceCube to be an efficient tool for neutrino astronomy over the next decade is limited by the modest numbers of cosmic neutrinos measured, even with a cubic 


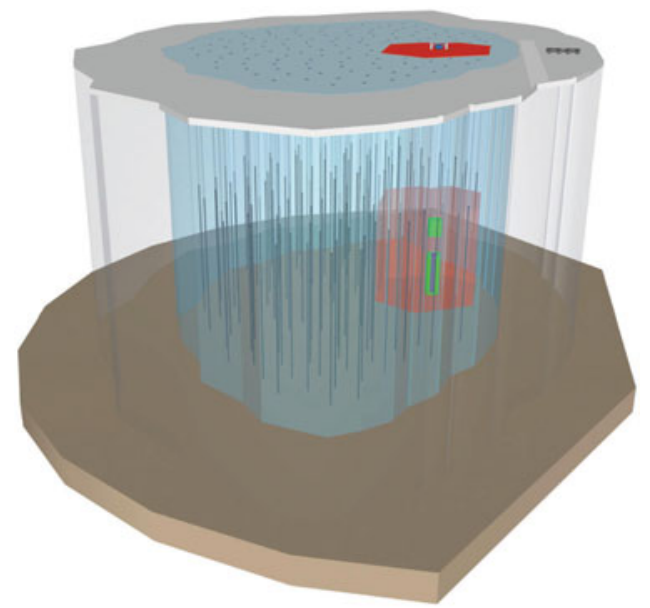

Figure 6. Conceptual schematic of the proposed high-energy extension to the IceCube detetector. The current IceCube is shown in red with DeepCore in green. 120 additional string are added to increase the instrumented volume to $\sim 10 \mathrm{~km}^{3}$. A veto detector comprised of scintillator detectors is also envisioned at the surface.
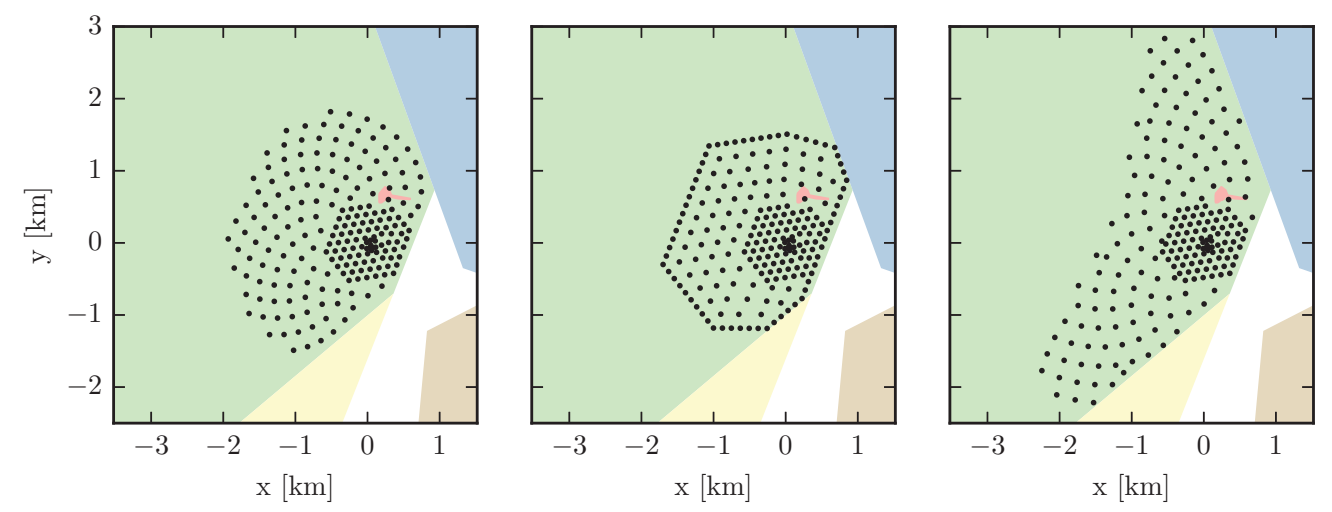

Figure 7. Example benchmark detector string layouts under study for the high energy extension to IceCube. Each expands about IceCube by adding 120 strings constrained to the South Pole Dark Sector (shaded in light green). For the left panel, uniform string spacing of $240 \mathrm{~m}$ is shown. The central panel represents a string layout with a denser edge weighting for improved veto efficiency. The right panel shows the so-called banana geometry which seeks to create a very long detector for certian muon tracks.

kilometer array. Design studies to increase IceCube's sensitivity with additional strings outside the current volume are currently underway (Aartsen et al. 2014a). This section will describe this effort, referred to as the IceCube-Gen2 High-Energy Array. The design, shown in Figure 6, seeks to increase the instrumented volume to $\sim 10 \mathrm{~km}^{3}$. The highenergy array is proposed to complement the high-density, low-energy sub-array known as PINGU (Aartsen et al. 2014b). PINGU targets precision measurements of the atmospheric oscillation parameters and the determination of the neutrino mass hierarchy.

The light properties of deep Antarctic ice allow string spacing to be increased to $300 \mathrm{~m}$ for energies exceeding $10 \mathrm{TeV}$. Since angular resolution for muon tracks is porportional to the length of the lever arm, by increasing the size of the detector, the angular resolution will also be improved, further improving point-source sensitivity. Studies to find the optimum geometry and string spacing are currently underway, some of the geometries 
can be seen in Figure 7. All of the designs add 120 strings to the detector within the region of the South Pole station designated as the dark sector. Uniform string spacings of $200 \mathrm{~m}, 240 \mathrm{~m}$, and $300 \mathrm{~m}$, which instrument volumes of $6.0 \mathrm{~km}^{3}, 8.0 \mathrm{~km}^{3}$, and $11.9 \mathrm{~km}^{3}$ respectively, have been studied. Alternative array designs are also under study. The edge weighted geometry was conceived to improve the efficiency at which the outer layers aid in identifying interactions which occur inside the detector volume. It would have edge strings spaced at $125 \mathrm{~m}$, while interior strings would be spaced at $240 \mathrm{~m}$, with an instrumented volume of $6.2 \mathrm{~km}^{3}$. The so-called banana geometry would create one direction in which muon tracks would traverse a very long instrumented distance which will improve angular resolution along that axis. Tracks which traverse perpendicular to this direction would not benefit from the increased angular resolution but would see an increased detector size. In addition, IceCube-Gen2's reach may further be enhanced by exploiting the air-shower detection and vetoing capabilities of an extended surface array, or by including an extended $100 \mathrm{~km}^{2}$ radio array to achieve improved sensitivity to neutrinos in the $10^{16}-10^{20} \mathrm{eV}$ energy range, including GZK neutrinos.

While the design details remain to be finalized, IceCube-Gen2 will reveal an unobstructed view of the universe at $\mathrm{PeV}$ energies where most of the universe is opaque to high-energy photons. It will operate simultaneously with next generation of electromagnetic and gravitational wave detectors, allowing for more multimessenger analyses. With its unprecedented sensitivity and improved angular resolution, this instrument will enable detailed spectral studies, significant point source detections and new discoveries.

\section{References}

Aartsen, M. G. et al. 2014a, arXiv:1412.5106.

Aartsen, M. G. et al. 2014b, arXiv:1401.2046v1.

Aartsen, M. G. et al. 2015a, in: Proceedings of the 34 th International Cosmic Ray Conference. PoS(ICRC2015)1081. arXiv: 1510.05223v2.

Aartsen, M. G. et al. 2015b, in: Proceedings of the 34th International Cosmic Ray Conference. PoS(ICRC2015)1109. arXiv: 1510.05223v2.

Aartsen, M. G. et al. 2015c, ApJ 805 L5. arXiv:1412.6510

Aartsen, M. G. et al. 2015d, in: Proceedings of the 34th International Cosmic Ray Conference. PoS(ICRC2015) 1047. arXiv:1510.05222v3.

Aartsen, M. G. et al. 2016a, accepted for publication in ApJ. arXiv:1607.08006.

Aartsen, M. G. et al. 2016b, ApJ 824 115. arXiv:1601.06484.

Aartsen, M. G. et al. 2016c, submitted to J. Inst.

Abbasi, R. et al. 2012, A\&A 539 A60. arXiv:1111.7030.

Adrián-Martánez, S. et al. 2016, Phys. Rev. D 93, 122010. arXiv:1602.05411.

Ahlers, M., Gonzalez-Garcia, M., \& Halzen, F. 2011, APh 35, 87. arXiv:1103.3421

Enberg, R., Reno, M. H., \& Sarcevic, I. 2008, Phys.Rev.D 78, 043005. arXiv:0806. 0418.

Glüsenkamp, T. 2016, in: Roma International Conference on Astroparticle Physics 2014 (RICAP14), EPJ Web of Conferences Volume 121. arXiv:1502.03104.

Goldstein, A. et al. 2012, ApJS 199, 19. arXiv:1201.2981.

Katz, B., Budnik, R., \& Waxman, E. 2009, JCAP 2009, 020. arXiv:0811.3759

Waxman, E. \& Bahcall, J. 1997, PhRvL 78, 2292. astro-ph/9701231 